\section{Kidney Blood Pressure Research}

Kidney Blood Press Res 2018;43:1121-1130

DOI: $10.1159 / 000491808$

Published online: 16 July, 2018

Accepted: 5 July, 2018

This article is licensed under the Creative Commons Attribution-NonCommercial-NoDerivatives 4.0 International License (CC BY-NC-ND) (http//wwwkarger.com/Services/OpenAccessLicense). Usage and distribution for commercial purposes as well as any distribution of modified material requires written permission.

\title{
Association Between Thyroid-Stimulating Hormone and Renal Function: a Mendelian Randomization Study
}

\author{
Chi Chen Fangzhen Xia Yi Chen Kun Zhang Jing Cheng Qin Li Bing Han \\ Li Zhao Chunfang Zhu Ningjian Wang Yingli Lu
}

Institute and Department of Endocrinology and Metabolism, Shanghai Ninth People's Hospital, Shanghai JiaoTong University School of Medicine, Shanghai, China

\section{Key Words}

Thyroid-stimulating hormone $\bullet$ Estimated glomerular filtration rate $•$ Epidemiology $•$ Mendelian randomization

\begin{abstract}
Background/Aims: Increasing evidence suggests an association between thyroid-stimulating hormone (TSH) and estimated glomerular filtration rate (eGFR). We conducted a Mendelian randomization (MR) analysis to examine the causality of the association between TSH and eGFR. Methods: 10,603 participants were recruited from the Survey on Prevalence in East China for Metabolic Diseases and Risk Factors (SPECT-China), which was performed in 23 sites in East China during 2014-2016. We constructed weighted genetic risk scores (GRS) for TSH based on three TSH-related single nucleotide polymorphisms. eGFR was calculated using the CKD Epidemiology Collaboration formula. The instrumental variable (IV) was used to explore the causal relationship between TSH and eGFR. Results: Higher measured TSH levels were associated with lower eGFR (B -0.717, 95\%CI -0.958, -0.476) after multivariable adjustment. However, by MR analysis, per SD increase in the TSH_GRS was significantly associated with TSH (B $0.155,95 \%$ CI $0.076,0.235, P<0.001)$ but not with eGFR (B $-0.127,95 \% C I-0.364,0.110)$. Using IV estimator, no causal associations were observed for genetically instrumented TSH with eGFR. Conclusion: By a genetic approach that limits residual confounding and reverse causation in observational conventional epidemiological studies, TSH and eGFR are not causally associated, which suggests genetically elevated TSH concentrations may not affect the renal function.




\section{Kidney Blood Pressure Research}

Chen et al.: TSH and eGFR

\section{Introduction}

Chronic kidney disease (CKD) is a rising contributor to the global disease burden. CKD increases the risk of all-cause mortality, cardiovascular disease, and progression to endstage renal disease [1]. Now, approximately $13.1 \%$ of Americans and $10.8 \%$ of Chinese adult population are affected by CKD [2,3]. Although hypertension, diabetes mellitus, and obesity, etc., have been established as risk factors for CKD [4], identification of new risk factors for CKD, or decline in estimated glomerular filtration rate (eGFR), which is a surrogate for development of CKD, may help extensively understand the pathogenesis of CKD and develop novel prevention strategies for CKD.

Thyroid-stimulating hormone (TSH) might lower eGFR. Evidence from cross-sectional studies supports this speculation: high TSH was associated with low eGFR and increased odds ratio of CKD [5-7]. Prospective studies exploring the effect of thyroid hormones on renal function decline were limited and reported discrepant findings. One study found that high-normal TSH levels were associated with an increased risk of incident CKD in euthyroid individuals [8]. Other two studies found no association between TSH and change in renal function $[9,10]$. However, data from the Rotterdam Study reported that higher TSH levels were associated with less annual eGFR decline and lower CKD incidence [11]. Due to the nature of cross-sectional study and conflicting results of prospective studies, the causality between TSH and eGFR should be further confirmed.

An alternative to classical observational study is mendelian randomization (MR) study, which is not susceptible to confounding and reverse causality [12]. MR uses genetic variants in non-experimental data to derive estimates of the causal effect of an exposure on an outcome [13]. In this study, if high TSH is causally related to lower eGFR, genetic variants associated with higher TSH should be associated with lower eGFR. These genetic variants are inherited from parents to offspring during meiosis at conception and remained unchanged throughout life [14]. Thus, analogous to randomized clinical trials, MR could overcome limitations in conventional epidemiological studies such as reverse causation and residual confounding [13].

In the present study, we set out to use a MR approach to investigate the causal association between TSH and eGFR based on the large community-based sample from SPECT-China study (Survey on Prevalence in East China for metabolic diseases and risk factors). TSH genetic risk score (TSH_GRS) was constructed to represent the genetic susceptibility.

\section{Materials and Methods}

\section{Participants}

The data were from the ongoing SPECT-China study, which is a large cross-sectional study (ChiCTRECS-14005052, www.chictr.org.cn). Recruitment and enrollment have been described in detail in previous papers [15-17]. We included Chinese citizens $\geq 18$ years old who had lived in their current area for $\geq 6$ months and excluded subjects with severe communication problems, acute illness or who were unwilling to participate. From 2014 to 2016, 12666 subjects who were 18-93 years old were recruited in the SPECT-China study from 23 sites in Shanghai, Zhejiang, Jiangsu, Anhui and Jiangxi Province. Among them, genotype information was available in 10, 664 participants (84.2\%). We excluded the participants who missed information on TSH ( $\mathrm{n}=40)$ or eGFR $(\mathrm{n}=2)$ or more than two single nucleotide polymorphism (SNP) genotypes $(n=19)$. Finally, a total of 10,603 participants were involved in this analysis.

The Ethics Committee of Shanghai Ninth People's Hospital, Shanghai JiaoTong University School of Medicine approved the protocol [approval number 2013 (86)]. The study was conducted in accordance with the ethical standards of the responsible committee on human experimentation (institutional and national) and with the Helsinki Declaration of 1975, as revised in 2008. All participants gave informed consent before data collection. 


\section{Kidney Blood Pressure Research}

Chen et al.: TSH and eGFR

\section{Measurements}

Interview and collection of biological specimens were performed by the same trained staff at each site with a single assessment protocol. Blood samples were drawn between 7:00 am and 10:00 am after participants were fasted for at least $8 \mathrm{~h}$. Blood was refrigerated immediately after phlebotomy, and after 2-4h it was centrifugated and the serum was aliquoted and frozen in a central laboratory, which was certified by the College of American Pathologists. Serum total tetraiodothyronine (TT4), total triiodothyronine (TT3) and thyroid-stimulating hormone (TSH) were measured by the chemiluminescence immunoassay (SIEMENS immulite 2000, Germany). Glycated hemoglobin (HbA1c) was measured by high-performance liquid chromatography (MQ-2000PT, Medconn, Shanghai, China). Fasting plasma glucose (FPG) and serum creatinine were measured by a Beckman Coulter AU 680 (Brea, USA).

Body mass index (BMI) was calculated as weight $(\mathrm{kg})$ divided by the square of height $\left(\mathrm{m}^{2}\right)$. Current smoking was defined as having smoked at least 100 cigarettes in one's lifetime and currently smoking cigarettes [18]. Blood pressure was measured using standard methods [18].

eGFR was calculated using the following CKD-EPI formula: (1) for female whose Scr $\leq 0.7 \mathrm{mg} / \mathrm{dL}$, eGFR $\left(\mathrm{mL} / \mathrm{min} / 1.73 \mathrm{~m}^{2}\right)=144 \times(\mathrm{Scr} / 0.7)^{-0.329} \times(0.993)^{\text {age }}$; (2) for female whose Scr $>0.7 \mathrm{mg} / \mathrm{dL}$, eGFR $=144 \times$ $(\mathrm{Scr} / 0.7)^{-1.209} \times(0.993)^{\mathrm{age}}$; (3) for male whose $\mathrm{Scr} \leq 0.9 \mathrm{mg} / \mathrm{dL}, \mathrm{eGFR}=141 \times(\mathrm{Scr} / 0.9)^{-0.411} \times(0.993)^{\text {age }} ;(4)$ for male whose $\mathrm{Scr}>0.9 \mathrm{mg} / \mathrm{dL}, \mathrm{eGFR}=141 \times(\mathrm{Scr} / 0.9)^{-1.209} \times(0.993)^{\text {age }}$.

Diabetes was defined as a previous diagnosis by physicians, a FPG $\geq 7.0 \mathrm{mmol} / \mathrm{L}$, or a $\mathrm{HbA} 1 \mathrm{c} \geq 6.5 \%$. Hypertension was defined as a systolic blood pressure (SBP) of $140 \mathrm{mmHg}$ or higher, a diastolic blood pressure (DBP) of $90 \mathrm{mmHg}$ or higher, or self-reported previous diagnosis of hypertension by physicians.

Genotyping, genetic loci selection and genetic risk score construction

DNA was extracted from blood leukocytes using a blood genomic DNA extraction kit (OSR-M102-T1, TIANGEN BIOTECH CO, LTD, Beijing, China) on an automated nucleic acid extraction instrument (OSE-M48, TIANGEN BIOTECH CO, LTD, Beijing, China). Specific assays were designed using Geneious Pro (v4.8.3) (https://www.geneious.com/). Mass determination was carried out with the JUNO and data acquisition was completed using Fluidigm SNP Genotyping Analysis v4.1.3software (Fluidigm Corporation, South San Francisco, California, USA). Call rates of all included SNPs were greater than $98 \%$.

We identified 3 SNPs involved in susceptibility of TSH (XKR4rs2622590, CAPZBrs6683419, FOXE1rs925489) selected from a recently published genome wide association study (GWASs) in Chinese [19]. They all reached a genome-wide significance level $\left(P<5 \times 10^{-8}\right)$ and not in linkage disequilibrium $\left(r^{2}=0\right)$.

\section{Statistical analysis}

IBM SPSS Statistics, Version 24 was used for data analyses (IBM Corporation, Armonk, NY, USA). A two-sided $P$ value $<.05$ indicated significance. Continuous variables were presented as the mean \pm standard deviation (SD) and categorical variables as a percentage (\%), respectively.

Construction of GRS was performed using the additive genetic model for each SNP (coded as 0, 1, and 2 to genotypes containing 0, 1,or 2 risk alleles, respectively) [20]. For the TSH_GRS, we derived a weighted score by multiplying the number of TSH increasing alleles of each SNP with the respective $\beta$ coefficient from the literature [19] and then taking the sum of all values. The characteristics of the individual SNP in the TSH_GRS were shown in Table 1. Weighted GRS was used in the present study, and the un-weighted GRS was used in the sensitivity analysis.

We assessed the strength of associations using F statistics $\left[F=\left(R^{2} *(n-2)\right) /\left(1-R^{2}\right)\right][21]$, with values greater than 10 regarded as useful for mendelian randomization analysis [14].

Linear regression analyses were applied to determine the associations of the TSH_GRS and TSH with eGFR. Model 1 adjusted for age, sex and TT4. Model 2 adjusted for age, sex, TT4, BMI, current smoking (yes or no), hypertension (yes or no) and diabetes (yes or no).

Table 1. Information of each SNP in TSH_GRS. EAF, effect allele frequency; GRS, genetic risk score; HWE, Hardy-Weinberg equilibrium; SNP, single nucleotide polymorphism

\begin{tabular}{lccccccc}
\hline Gene & Chromosome & Position & SNP & Effect allele & EAF & $X^{2}$ & P for HWE \\
\hline CPAZB & 1 & 19827780 & rs6683419 & G & 0.28 & 0.94 & $>0.05$ \\
XKR4 & 8 & 56358274 & rs2622590 & G & 0.58 & 1.39 & $>0.05$ \\
FOXE1 & 9 & 100546600 & Rs925489 & T & 0.92 & 0.67 & $>0.05$ \\
\hline
\end{tabular}




\section{Kidney Blood Pressure Research}

Chen et al.: TSH and eGFR

Regarding the MR analysis, the weighted TSH_GRS was used as the instrumental variables (IV) estimators. The formal MR analyses to estimate the possible causal effect of TSH on eGFR were done using the IV ratio method [14]. For the decreased eGFR because of higher TSH, the computational formula was $\beta_{\text {IV(TSH-EGFR) }}=\beta_{\text {TSH_GRS-eGFR }} / \beta_{\text {TSH_GRS-TSH }}$ The model was adjusted for age, sex, TT4, BMI, current smoking (yes or no), hypertension (yes or no), and diabetes (yes or no). The standard error (SE) and confidence interval (CI) for the IV estimators was estimated by the delta method. The formulas were shown as below.

$$
\begin{aligned}
& \mathrm{SE}_{\mathrm{IV}}=\operatorname{abs}\left(\beta_{\mathrm{IV}}\right) \sqrt{\left(\frac{\mathrm{SE}_{\mathrm{GRS}_{-} \mathrm{TSH}}}{\beta_{\mathrm{GRS}_{-} \mathrm{TSH}}}\right)^{2}+\left(\frac{\mathrm{SE}_{\mathrm{GRS}_{\text {e }} \mathrm{GFR}}}{\beta_{\mathrm{GRS}_{\text {eGFR }}}}\right)^{2}} \\
& 95 \% \mathrm{CI}_{\mathrm{IV}}=\beta_{\mathrm{IV}} \pm 1.96 \times \mathrm{SE}_{\mathrm{IV}}
\end{aligned}
$$

To validate the genetic instruments, we assessed the associations between each individual SNP with TSH and eGFR, respectively. We also measured the potential pleotropic associations of each individual SNP and the GRS with BMI, FPG, HbA1c, SBP and DBP.

In sensitivity analysis, we derived the unweighted TSH_GRS and TSH_GRS ${ }_{2 S N P}$ removing the SNP may have pleiotropic effect on eGFR-related metabolic risk factors. Then we used them as the IVs.

\section{Results}

\section{Association between SNPs with TSH}

We tested the association between three TSH-related SNPs with TSH. Unstandardized coefficients $(95 \%$ confidence interval (CI)) of additive regression models are shown in Fig. 1. In the three TSH-related SNPs, two SNPs located at the CPAZB or FOXE1 loci were significantly associated with TSH in this study. The other SNP located at the XKR4 loci also showed a marginal significance with TSH $(P=0.08)$.

\section{Pleiotropic effects of SNPS}

We checked whether the SNPs showed any association with TSH and CKD related major metabolic traits. Thus, we tested the potential associations of the SNPs with BMI, FPG, HbA1c, SBP and DBP using an additive model. The results are presented in Table 2 . Except for the association between rs6683419 and $\mathrm{HbA1C}$, we did not identify any significant association of TSH genetic instrument with BMI, FPG, HbA1c, SBP and DBP.

\section{Characteristics of study participants} according to TSH_GRS Quartiles

As expected, with the increasing $\mathrm{TSH}_{-}$ GRS, serum TSH concentrations gradually and markedly increased. However, the TSH_GRS was neither consistently associated with TT3 and TT4, nor with the risk factors such as age, sex, smoking status, hypertension or BMI (Table 3). There was no trend between $\mathrm{TSH}_{-}$ GRS and eGFR.

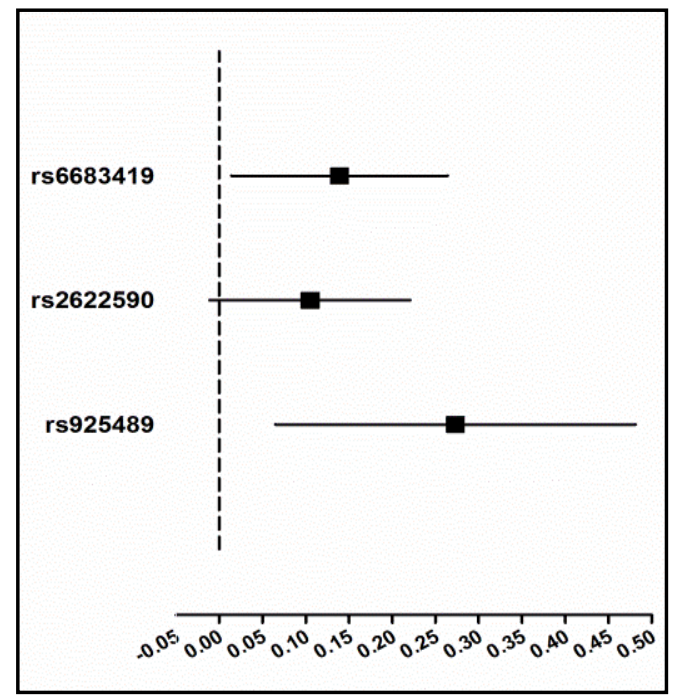

Fig. 1. Association of the three TSH-related SNPS with TSH. Linear regression analyses were used and the model was adjusted for age, sex and BMI. Solid squares represented unstandardized coefficients of each individual SNP with TSH. The 95\% confidence intervals were denoted by lines. 


\section{Kidney \\ Blood Pressure Research}

Chen et al.: TSH and eGFR

Table 2. The association between each individual SNP with metabolic confounders. Data are expressed as unstandardized coefficients (standard error). BMI, body mass index; FPG, fasting plasma glucose; HbA1C, glycated hemoglobin; SBP, systolic blood pressure; DBP, diastolic blood pressure

\begin{tabular}{|c|c|c|c|c|c|c|c|c|c|c|}
\hline SNP & BMI & & FPG & & $\mathrm{HbA} 1 \mathrm{C}$ & & SBP & & DBP & \\
\hline & $\mathrm{B}(\mathrm{SE})$ & $\mathrm{P}$ & $\mathrm{B}(\mathrm{SE})$ & $\mathrm{P}$ & $\mathrm{B}(\mathrm{SE})$ & $\mathrm{P}$ & $\mathrm{B}(\mathrm{SE})$ & $P$ & $\mathrm{~B}(\mathrm{SE})$ & $\mathrm{P}$ \\
\hline rs668 & $-0.05(0.06)$ & 0.41 & $-0.02(0.02)$ & 0.34 & $-0.03(0.01)$ & 0.02 & $-0.14(0.30)$ & 0.64 & $-0.02(0.19)$ & 0.91 \\
\hline rs2622590 & $-0.05(0.05)$ & 0.36 & $-0.01(0.02)$ & 0.53 & $0.01(0.01)$ & 0.43 & $0.26(0.28)$ & 0.34 & $0.15(0.18)$ & 0.41 \\
\hline rs925489 & $-0.03(0.09)$ & 0.08 & $0.02(0.04)$ & 0.56 & $<0.01(0.02)$ & 0.99 & $-0.70(0.50)$ & 0.16 & $-0.37(0.32)$ & 0.24 \\
\hline
\end{tabular}

Table 3. Characteristics of study participants according to the weighted TSH genetic risk score (TSH_ GRS). The data are summarized as the mean (SD) for continuous variables or as a numerical proportion for categorical variables. P for trend was calculated by ANOVA and chi-square tests. Since TSH was nonnormally distributed, they were log-transformed. TSH, thyroid-stimulating hormone; GRS, genetic risk score; TT3, total triiodothyronine; TT4, total thyroxine; BMI, body mass index; eGFR, estimated glomerular filtration rate

\begin{tabular}{|c|c|c|c|c|c|}
\hline \multirow{2}{*}{ Variable } & \multicolumn{4}{|c|}{ TSH_GRS } & \multirow{2}{*}{ P for trend } \\
\hline & Q1 & Q2 & Q3 & Q4 & \\
\hline TSH_GRS & $\leq 0.186$ & $0.187-0.193$ & $0.194-0.226$ & $\geq 0.227$ & \\
\hline $\mathrm{N}$ & 2121 & 3180 & 3380 & 1859 & \\
\hline Age, yr & $55(13)$ & $55(13)$ & $55(13)$ & $54(13)$ & 0.409 \\
\hline Men, \% & 38.9 & 39.1 & 40.7 & 41.0 & 0.076 \\
\hline TSH, mIU/L & $0.32(0.30)$ & $0.35(0.30)$ & $0.35(0.32)$ & $0.38(0.29)$ & $<0.001$ \\
\hline TT4, nmol/L & $114.4(22.2)$ & $114.9(21.5)$ & $115.2(21.3)$ & $114.4(21.4)$ & 0.881 \\
\hline TT3, nmol/L & $1.79(0.45)$ & $1.78(0.43)$ & $1.77(0.43)$ & $1.78(0.44)$ & 0.632 \\
\hline BMI, $\mathrm{kg} / \mathrm{m}^{2}$ & $24.8(3.7)$ & $24.5(3.6)$ & $24.6(3.6)$ & $24.6(3.4)$ & 0.180 \\
\hline Current smoker, \% & 19.8 & 19.5 & 21.1 & 20.4 & 0.287 \\
\hline Diabetes, $\%$ & 15.0 & 15.1 & 15.4 & 12.1 & 0.033 \\
\hline Hypertension, \% & 49.4 & 46.8 & 48.5 & 47.8 & 0.626 \\
\hline $\mathrm{eGFR}, \mathrm{ml} / \mathrm{min} / 1.73 \mathrm{~m}^{2}$ & $87.1(15.3)$ & $87.1(15.2)$ & $86.6(15.8)$ & $87.1(15.1)$ & 0.712 \\
\hline
\end{tabular}

\section{Association of TSH_GRS and TSH with eGFR}

First, in this cross-sectional study, 1 SD increase of TSH was negatively and significantly associated with eGFR after adjustment for age, sex and TT4 (B -0.781', 95\%CI -1.015, -0.546) (Table 4, Model 1). Further adjusting for other metabolic profiles slightly attenuated the association, but it remained significant (B -0.717', 95\%CI -0.958, -0.476).

Next, we measured the association of TSH_GRS with eGFR. In Table 4, per SD increase in TSH_GRS was not significantly associated with eGFR after adjustment for age, sex and TT4 (B -0.163, 95\%CI -0.395, 0.069) (Model 1). Further adjusting for BMI, current smoking (yes or no), hypertension (yes or no) and diabetes (yes or no) did not change the results (B $-0.127,95 \%$ CI $-0.364,0.110$ ) (Model 2). The quartiles of TSH_GRS also showed no significant association with eGFR.

Fig. 2 presents the association of genetically determined TSH with eGFR. In the IV analysis, the causal regression coefficient of genetically determined TSH for eGFR was -0.804 (95\% CI -2.364, 0.756).

\section{Sensitivity analysis}

There was one TSH-related SNP (rs6683419) having pleiotropic effects with HbA1C, though the weighted GRS was not associated with most metabolic traits expect for diabetes (Table 5). Thus, we excluded this SNP to construct TSH_GRS ${ }_{2 S N P}$, which is expected, was not associated with diabetes or other metabolic traits. The IV estimate for causal relationship from TSH to eGFR was -1.500 (95\%CI -3.456, 0.456). Moreover, using the unweighted GRSs, the results were similar as those using weighted GRSs. 


\section{Kidney \\ Blood Pressure Research}

Table 4. The associations of TSH_GRS and TSH with eGFR. Data were presented as B coefficients and $95 \%$ confidence interval. ${ }^{* * *} \mathrm{P}<0.001$. TSH, thyroid-stimulating hormone; GRS, genetic risk score; eGFR, estimated glomerular filtration rate. Model 1 adjusted for age, sex and TT4; Model 2 adjusted for terms in model 1, BMI, current smoking (yes or no), hypertension (yes or no) and diabetes (yes or no)

\begin{tabular}{lcc}
\hline & Model 1 & Model 2 \\
\hline Per SD increase of TSH_GRS & $-0.163(-0.395,0.069)$ & $-0.127(-0.364,0.110)$ \\
& & $1.00($ Ref.) \\
Quartiles of TSH_GRS & 1.00 (Ref.) & $-0.121(-0.801,0.559)$ \\
Q1 & $-0.105(-0.772,0.562)$ & $-0.373(-1.048,0.302)$ \\
Q2 & $-0.454(-1.116,0.207)$ & $-0.230(-1.004,0.544)$ \\
Q3 & $-0.308(-1.068,0.451)$ & 0.373
\end{tabular}

Per SD increase of TSH

Fig. 2. Instrumental variable (IV) estimated association between TSH and eGFR. Data were adjusted for age, sex, TT4, BMI, current smoking, hypertension and diabetes.

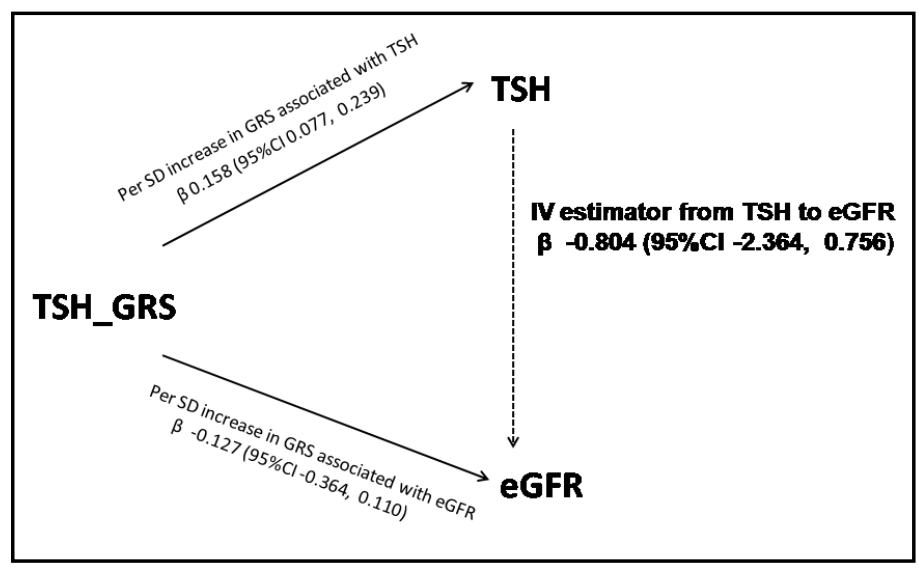

Table 5. Association of each 1-SD of the TSH_GRS with metabolic traits. Data are expressed as unstandardized coefficients (standard error) and odds ratio (95\% confidence interval). Multiple linear and logistic regression wasperformed. The model was adjusted for age, sex and BMI. TSH, thyroid-stimulating hormone; BMI, body mass index; FPG, fasting plasma glucose; HbA1C, glycated hemoglobin; SBP, systolic blood pressure; DBP, diastolic blood pressure

\begin{tabular}{|c|c|c|c|c|c|c|}
\hline Variable & TSH_GRS & $\mathrm{P}$ & TSH_GRS2SNP & $\mathrm{P}$ & TSH_GRS unweighted & $\mathrm{P}$ \\
\hline Continuous variable & B (SE) & & B (SE) & & $\mathrm{B}(\mathrm{SE})$ & \\
\hline TSH & $0.16(0.04)$ & $<0.01$ & $0.13(0.04)$ & $<0.01$ & $0.150 .04)$ & $<0.01$ \\
\hline BMI & $-0.04(0.04)$ & 0.22 & $-0.03(0.04)$ & 0.37 & $-0.05(0.04)$ & 0.20 \\
\hline FPG & $-0.01(0.01)$ & 0.60 & $<0.01(0.01)$ & 0.99 & $-0.01(0.01)$ & 0.42 \\
\hline $\mathrm{HbA1C}$ & $-0.01(0.01)$ & 0.42 & $0.01(0.01)$ & 0.59 & $-0.01(0.01)$ & 0.35 \\
\hline SBP & $-0.11(0.19)$ & 0.57 & $-0.07(0.19)$ & 0.71 & $-0.04(0.19)$ & 0.86 \\
\hline DBP & $-0.04(0.12)$ & 0.76 & $-0.03(0.12)$ & 0.78 & $0.01(0.12)$ & 0.96 \\
\hline Categorical variable & OR $(95 \% \mathrm{CI})$ & & & & OR $(95 \% \mathrm{CI})$ & \\
\hline Current smoking & $0.995(0.939,1.054)$ & 0.87 & $0.985(0.930,1.044)$ & 0.62 & $1.010(0.953,1.070)$ & 0.75 \\
\hline Diabetes & $0.944(0.892,0.998)$ & 0.04 & $0.981(0.927,1.037)$ & 0.49 & $0.934(0.882,0.988)$ & 0.02 \\
\hline Hypertension & $0.986(0.944,1.030)$ & 0.53 & $0.991(0.949,1.036)$ & 0.69 & $0.993(0.951,1.038)$ & 0.76 \\
\hline
\end{tabular}

\section{Discussion}

In this community-dwelling sample of Chinese adults, inverse cross-sectional associations between TSH and renal function were observed after multivariate adjustment. However, the results of MR analysis provide no evidence for a causal effect of high TSH on lowering eGFR. To date and to the best of our knowledge, this is the first study to explore the association between genetic variants associated with TSH and eGFR using the MR approach. 


\section{Kidney Blood Pressure Research}

Most, predominantly cross-sectional studies to date have focused on the hypotheses that hypothyroidism gives rise to several cardiovascular risk factors and circulatory changes through which kidney function could be affected [5-8]. Nevertheless, the lack of a genetically determined causal association between thyroid function and kidney function suggests that the previously observed association between TSH and eGFR might be explained by reverse causality or residual confounding. In case of reverse causality, evidence has shown that severe CKD commonly causes a hypothyroid state that exists in the absence of primary hypothalamic-pituitary-thyroid axis dysfunction [22, 23]. Moreover, unmeasured or unknown factors not taken into account as confounding variables in the observational studies might have affected the observed association between TSH and eGFR. Of note, although some intervention studies found L-thyroxine replacement therapy attenuated the rate of decline in renal function in CKD patients with hypothyroidism [24-26], the sample sizes were relatively small and none of these were randomized control trials.

In MR, genetic variant(s) are used as IVs to explore the causal effect of the exposure on the outcome. In our study, the three TSH SNPs were combined into a GRS. GRS is a convenient way of summarizing multiple genetic variants associated with an exposure of interest [27]. Since one SNP explains very little of a trait variance, using a GRS as IV instead of each individual SNP helps increase power and make stronger instruments [14]. To be a valid IV, GRS should fulfill three assumptions [14, 28]. First, the GRS should be associated with the exposure. All SNPs used in this study have previously been shown to be significantly associated with TSH in large GWAS. In the present study, the association of each SNP and the TSH_GRS with the TSH were also very significant. Second, the GRS should not be correlated with any confounders of the exposure-outcome relation. In our study, we found the $\mathrm{TSH}_{-}$ GRS were not associated with smoking status and most metabolic traits such as BMI and hypertension, which were common potential confounders of the TSH-eGFR association. We further tested the pleiotropic effects of each SNP on the above confounders and the results showed one SNP had pleiotropic effects (rs6683419) with HbA1C. Thus we removed this SNP and created the TSH_GRS ${ }_{2 S N P}$, but the IV estimate was not significantly changed from that in TSH_GRS ${ }_{3 S N P}$ and TSH_GRS ${ }_{2 S N P}$ were not associated with diabetes any more. Third, the GRS is independent of the outcome, except possibly via its influence on the exposure. It means that the only causal route from the genetic instrument to the outcome is through the exposure (no other routes between $\mathrm{TSH}_{-}$ GRS and eGFR) [28]. Thus, we analyzed the association of each SNP and TSH_GRS with the eGFR. Except rs2622590, the SNPs and GRS were not significantly associated with eGFR (Fig. 3). Further studies are required to gain more information about this SNP and its effect on eGFR beyond the effects through TSH.

The strength of our study was its welldefined community-based population, a relative large sample size, the MR study design and a created TSH_GRS representing the combined effect of the established common genetic variations of TSH as the IV. In addition, we carefully performed adjustments for potential confounders, including TT4. However, there are several limitations we should acknowledge. Firstly, although the association between TSH_GRS and TSH was statistically significant, the effect size was quite small (the

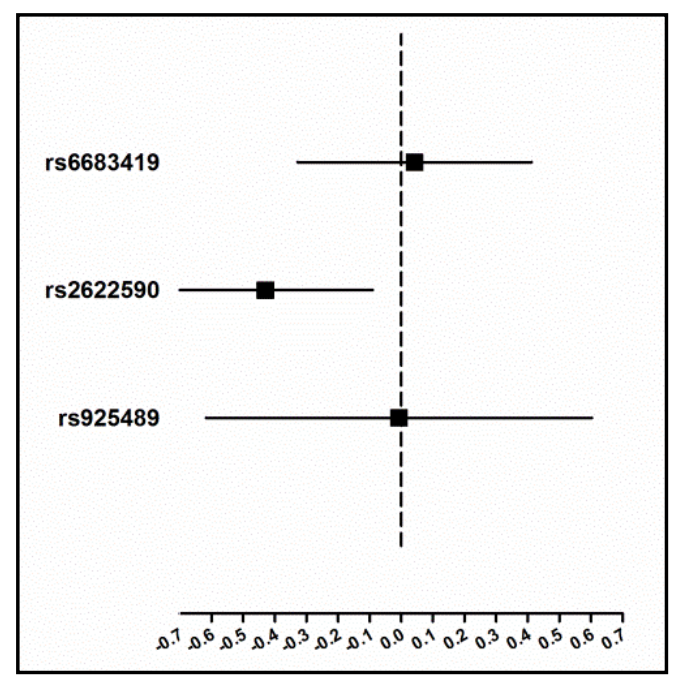

Fig. 3. Association of the three TSH-related SNPs with eGFR. Linear regression analyses were used and the model was adjusted for age, sex and BMI. Solid squares represented unstandardized coefficients of each individual SNP with eGFR. The $95 \%$ confidence intervals were denoted by lines. 


\section{Kidney \\ Blood Pressure Research}

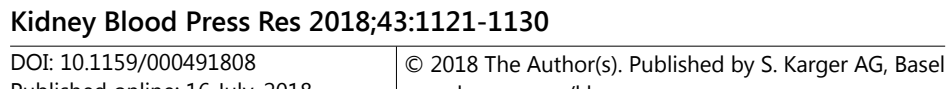

Published online: 16 July, 2018 www.karger.com/kbr

Chen et al.: TSH and eGFR

TSH increased from 0.32 to 0.38 from Q1 to Q4). This was because we created our TSH_GRS only on the basis of common variants, which was considered to represent limited amount of variance in TSH. We were unable to assess the potential contribution of rare variants. Future genetic-epidemiological studies with larger number of SNPs are required to validate our finding. Secondly, since we primarily used creatinine-based estimating equations for eGFR, we cannot rule out effects of thyroid hormones on the muscle metabolism of creatinine or its tubular secretion. Finally, we created the TSH_GRS by using the common variants that were robustly associated with TSH in East Asians, which may not be generalizable to other ethnic groups.

\section{Conclusion}

Although the concentration of TSH was associated with eGFR in cross-sectional study, we did not find a higher TSH_GRS was associated with lower eGFR by MR analysis. Our study suggested small variations in TSH are unlikely to be causative of eGFR variations; however, greater variations might still be causative and should be studied in a different setting. Further prospective cohort and multi-ethnicity MR studies with larger number of SNPs should be performed in subjects with all eGFR categories to allow reliable confirmation or refutation of the causal TSH_GFR relationships.

\section{Acknowledgements}

This study was supported by National Natural Science Foundation of China (81570726, 81600609); Shanghai JiaoTong University School of Medicine (2014); Science and Technology Commission of Shanghai Municipality (16410723200, 16411971200); Shanghai Municipal Commission of Health and Family Planning (2017YQ053); Commission of Health and Family Planning of Pudong District (PW2015D-5); The Fourth Round of Three-year Public Health Action Plan of Shanghai (15GWZK0202).

\section{Disclosure Statement}

The authors have no conflicts of interest regarding the publication of this paper.

\section{References}

1 Matsushita K, van der Velde M, Astor BC, Woodward M, Levey AS, de Jong PE, Coresh J, Gansevoort RT: Association of estimated glomerular filtration rate and albuminuria with all-cause and cardiovascular mortality in general population cohorts: A collaborative meta-analysis. Lancet 2010;375:2073-2081.

-2 Coresh J, Selvin E, Stevens LA, Manzi J, Kusek JW, Eggers P, Van Lente F, Levey AS: Prevalence of chronic kidney disease in the united states. JAMA 2007;298:2038-2047.

-3 Zhang L, Wang F, Wang L, Wang W, Liu B, Liu J, Chen M, He Q, Liao Y, Yu X, Chen N, Zhang JE, Hu Z, Liu F, Hong D, Ma L, Liu H, Zhou X, Chen J, Pan L, et al.: Prevalence of chronic kidney disease in China: a crosssectional survey. Lancet 2012;379:815-822.

-4 Jha V, Garcia-Garcia G, Iseki K, Li Z, Naicker S, Plattner B, Saran R, Wang AY, Yang CW: Chronic kidney disease: Global dimension and perspectives. Lancet 2013;382:260-272.

5 Chonchol M, Lippi G, Salvagno G, Zoppini G, Muggeo M, Targher G: Prevalence of subclinical hypothyroidism in patients with chronic kidney disease. Clin J Am Soc Nephrol 2008;3:1296-1300.

-6 Asvold BO, Bjoro T, Vatten LJ: Association of thyroid function with estimated glomerular filtration rate in a population-based study: The hunt study. Eur J Endocrinol 2011;164:101-105. 


\section{Kidney \\ Blood Pressure Research}

\begin{tabular}{l}
\hline Kidney Blood Press Res 2018;43:1121-1130 \\
\begin{tabular}{l|l}
\hline DOI: 10.1159/000491808 & (c) 2018 The Author(s). Published by S. Karger AG, Basel \\
Published online: 16 July, 2018 & www.karger.com/kbr
\end{tabular}
\end{tabular}

Published online: 16 July, 2018 www.karger.com/kbr

Chen et al.: TSH and eGFR

7 Peixoto de Miranda EJ, Bittencourt MS, Goulart AC, Santos IS, de Oliveira Titan SM, Ladeira RM, Barreto SM, Lotufo PA, Bensenor IJ: Thyrotropin levels are associated with chronic kidney disease among healthy subjects in cross-sectional analysis of the brazilian longitudinal study of adult health (elsa-brasil). Clin Exp Nephrol 2017;21:1035-1043.

-8 Zhang Y, Chang Y, Ryu S, Cho J, Lee WY, Rhee EJ, Kwon MJ, Pastor-Barriuso R, Rampal S, Han WK, Shin H, Guallar E: Thyroid hormone levels and incident chronic kidney disease in euthyroid individuals: The kangbuk samsung health study. Int J Epidemiol 2014;43:1624-1632.

-9 Meuwese CL, Gussekloo J, de Craen AJ, Dekker FW, den Elzen WP: Thyroid status and renal function in older persons in the general population. J Clin Endocrinol Metab 2014;99:2689-2696.

10 Huang X, Ding L, Peng K, Lin L, Wang T, Zhao Z, Xu Y, Lu J, Chen Y, Wang W, Bi Y, Ning G, Xu M: Thyroid hormones associate with risk of incident chronic kidney disease and rapid decline in renal function: A prospective investigation. J Transl Med 2016;14:336.

-11 Chaker L, Sedaghat S, Hoorn EJ, Elzen WP, Gussekloo J, Hofman A, Ikram MA, Franco OH, Dehghan A, Peeters RP: The association of thyroid function and the risk of kidney function decline: A population-based cohort study. Eur J Endocrinol 2016;175:653-660.

12 Sekula P, Del Greco MF, Pattaro C, Köttgen A: Mendelian randomization as an approach to assess causality using observational data. J Am Soc Nephrol 2016;27:3253-3265.

13 Smith GD, Ebrahim S: 'Mendelian randomization': Can genetic epidemiology contribute to understanding environmental determinants of disease? Int J Epidemiol 2003;32:1-22.

14 Lawlor DA, Harbord RM, Sterne JA, Timpson N, Davey Smith G: Mendelian randomization: Using genes as instruments for making causal inferences in epidemiology. Stat Med 2008;27:1133-1163.

15 Wang N, Wang X, Han B, Li Q, Chen Y, Zhu C, Chen Y, Xia F, Cang Z, Zhu C, Lu M, Meng Y, Chen C, Lin D, Wang $B$, Jensen MD, Lu Y: Is exposure to famine in childhood and economic development in adulthood associated with diabetes? J Clin Endocrinol Metab 2015;100:4514-4523.

16 Chen C, Xu H, Chen Y, Chen Y, Li Q, Hu J, Liang W, Cheng J, Xia F, Wang C, Han B, Zheng Y, Jiang B, Wang $\mathrm{N}$, Lu Y: Iodized salt intake and its association with urinary iodine, thyroid peroxidase antibodies, and thyroglobulin antibodies among urban chinese. Thyroid 2017;27:1566-1573.

17 Zhai H, Chen C, Wang N, Chen Y, Nie X, Han B, Li Q, Xia F, Lu Y: Blood lead level is associated with nonalcoholic fatty liver disease in the yangtze river delta region of china in the context of rapid urbanization. Environ Health 2017;16:93.

18 Xu Y, Wang L, He J, Bi Y, Li M, Wang T, Wang L, Jiang Y, Dai M, Lu J, Xu M, Li Y, Hu N, Li J, Mi S, Chen CS, Li G, Mu Y, Zhao J, Kong L, et al.: Prevalence and control of diabetes in chinese adults. JAMA 2013;310:948-959.

19 Zhan M, Chen G, Pan CM, Gu ZH, Zhao SX, Liu W, Wang HN, Ye XP, Xie HJ, Yu SS, Liang J, Gao GQ, Yuan GY, Zhang XM, Zuo CL, Su B, Huang W, Ning G, Chen SJ, Chen JL, et al.: Genome-wide association study identifies a novel susceptibility gene for serum tsh levels in chinese populations. Hum Mol Genet 2014;23:55055517.

20 Palmer TM, Lawlor DA, Harbord RM, Sheehan NA, Tobias JH, Timpson NJ, Davey Smith G, Sterne JA: Using multiple genetic variants as instrumental variables for modifiable risk factors. Stat Methods Med Res 2012;21:223-242.

-21 Vimaleswaran KS, Berry DJ, Lu C, Tikkanen E, Pilz S, Hiraki LT, Cooper JD, Dastani Z, Li R, Houston DK, Wood AR, Michaelsson K, Vandenput L, Zgaga L, Yerges-Armstrong LM, McCarthy MI, Dupuis J, Kaakinen M, Kleber ME, Jameson K, et al.: Causal relationship between obesity and vitamin d status: Bi-directional mendelian randomization analysis of multiple cohorts. PLoS medicine 2013;10:e1001383.

22 Kaptein EM: Thyroid hormone metabolism and thyroid diseases in chronic renal failure. Endocr Rev 1996;17:45-63.

23 Sanai T, Okamura K, Rikitake S, Fukuda M, Onozawa K, Sanematsu M, Takashima T, Miyazono M, Ikeda Y: The high prevalence of reversible subclinical hypothyroidism with elevated serum thyroglobulin levels in chronic kidney disease patients. Clin Nephrol 2017;87:237-244.

24 Shin DH, Lee MJ, Lee HS, Oh HJ, Ko KI, Kim CH, Doh FM, Koo HM, Kim HR, Han JH, Park JT, Han SH, Yoo TH, Kang SW: Thyroid hormone replacement therapy attenuates the decline of renal function in chronic kidney disease patients with subclinical hypothyroidism. Thyroid 2013;23:654-661. 


\section{Kidney

25 Hataya Y, Igarashi S, Yamashita T, Komatsu Y: Thyroid hormone replacement therapy for primary hypothyroidism leads to significant improvement of renal function in chronic kidney disease patients. Clin Exp Nephrol 2013;17:525-531.

26 Lu Y, Guo H, Liu D, Zhao Z: Preservation of renal function by thyroid hormone replacement in elderly persons with subclinical hypothyroidism. Arch Med Sci 2016;12:772-777.

-27 Burgess S, Thompson SG: Use of allele scores as instrumental variables for mendelian randomization. Int J Epidemiol 2013;42:1134-1144.

28 Sheehan NA, Didelez V, Burton PR, Tobin MD: Mendelian randomisation and causal inference in observational epidemiology. PLoS medicine 2008;5:e177. 